\section{Optical Amplification Achieved in a First-Generation Dendritic Organic Semiconductor}

Since the discovery of their light-emitting properties, organic semiconductors have stimulated great interest within the display industries and opened opportunities for other optoelectronic devices, such as visible laser diodes and, most recently, optical amplifiers.

There are three main classes of lightemitting organic semiconductors, of which the youngest technology is conjugated dendrimers - a family of highly branched molecules. In a dendrimer, the molecular branches form a snowflakelike structure that converges to a single focal point or core. This type of structure allows for the independent tuning of the electrical, optical, and processing properties of the material.

As reported in the April 15 issue of Optics Letters, researchers J.R. Lawrence of St. Andrews University, G.J. Richards of Oxford University, and their colleagues have observed strong optical amplification of violet light in both the liquid and solid states of first-generation bis(fluorene)cored semiconducting dendrimers. The core, consisting of a bis(fluorene) unit

\section{Review Articles and Special Issues}

Journal of Physical and Chemical Reference Data 33 (2) (June 2004) contains A. Jablonski, F. Salvat, and C.J. Powell, "Comparison of Electron Elastic-Scattering Cross Sections Calculated from Two Commonly Used Atomic Potentials," p. 409; and S.K. Mishra, R.K.S. Yadav, V.B. Singh, and S.B. Rai, "Spectroscopic Studies of Diatomic Indium Halides," p. 453.

Journal of Applied Physics 95 (8) (April 2004) contains C.G. Van de Walle and J. Neugebauer, "First-Principles Calculations for Defects and Impurities: Applications to III-Nitrides," p. 3851.

Journal of Environmental Engineering 130 (4) (April 2004) contains the Simon W. Freese Environmental and Engineering Lecture by P. Sarin, V.L. Snoeyink, D.A. Lytle, and W.M. Kriven, "Iron Corrosion Scales: Model for Scale Growth, Iron Release, and Colored Water Formation," p. 364.

Reviews of Scientific Instruments 75 (4) (April 2004) contains R.S. Lakes, "Viscoelastic Measurement Techniques," p. 797.

Low Temperature Physics 30 (3) (March 2004) contains V.M. Loktev and V.M. Turkowski, "Doping-Dependent Superconducting Properties of TwoDimensional Metals with Different Types of Interparticle Coupling," p. 179.

Physics of the Solid State 46 (3) (March 2004) contains A.I. Morosov and A.S. Sigov, "New Type of Domain Walls: Domain Walls Caused by Frustrations in Multilayer Magnetic Nanostructures," p. 395.

Solid State Electronics 48 (6) (June 2004) is a special issue on Silicon-on-Insulator Technology and Devices.

Journal of Materials in Civil Engineering 16 (2) (April2004) is a special issue on Micromechanical Characterization and Constitutive Modeling of Asphalt Mixes.

Journal of Microlithography, Microfabrication, and Microsystems 3 (2) (April 2004) contains a special section on Mask Technology for Optical Lithography.

IEEE Transactions on Nanobioscience 3 (1) (March 2004) is a special issue on Nanocapsules: A European Community Interdisciplinary Network in the Nanobiosciences.

Journal of Biomedical Optics 9 (2) (March, 2004) contains a special section on the Optics of Human Skin.

IEEE Journal of Solid State Circuits 39 (4) (April 2004) contains papers from the 2003 Symposium on VLSI Circuits held in Kyoto, Japan, June 12-14, 2003.

Thin Solid Films (April 2004) contains the proceedings of Symposium H on Photonic Processing of Surfaces, Thin Films, and Devices from the 2003 E-MRS Spring Conference held in Strasbourg, France, June 10-13, 2003.

Vacuum 73 (3-4) (April 19, 2004) contains the proceedings of the 4 th International Symposium on Applied Plasma Science, held in Kyoto, Japan, September 1-5, 2003.

IEEE Transactions on Nanotechnology 3 (1) (March 2004) contains papers from the Second International Workshop on Quantum Dots for Quantum Computing and Classical Size Effect Circuits (IWQDQC-2), held in Notre Dame, Indiana, August 7-9, 2003.

Vacuum 73 (2) (March 19, 2004) contains the proceedings of the European Vacuum Congress Berlin 2003, featuring the 8th European Vacuum Conference, 2nd Annual Conference of the German Vacuum Society, June 23-26, 2003. capped with two first-generation triphenyl-based dendrons containing 2-ethylhexyloxy surface groups, was made by using palladium catalysis to couple the first-generation boronic-acid-focused dendron with 7,7' dibromobisfluorene. The liquid optical amplifier was based upon a solution of dendrimer mixed with toluene. The solid-state optical amplifier was made by spin-coating the dendrimer solution onto quartz substrates to produce slab waveguides in 200-nm-thick films. The researchers used a $337 \mathrm{~nm}$ wavelength, pulsed nitrogen laser as the excitation source and observed optical gains of $36 \mathrm{~dB}$ $\mathrm{cm}^{-1}$ and $26 \mathrm{~dB} \mathrm{~cm}^{-1}$ at $429 \mathrm{~nm}$ and $390 \mathrm{~nm}$, respectively, in solution, and a maximum gain of $350 \mathrm{~dB} \mathrm{~cm}^{-1}$ at $425 \mathrm{~nm}$ in the solid state. These results compare well with values reported for other organic semiconducting materials. The researchers believe that dendrimers offer considerable scope for molecular designers to tune the gain across the visible spectrum, and that the solid-state form has potential for electrically pumped amplification.

SHIMING Wu

\section{Nanopatterning on a Biocompatible Polymer Film Accomplished with UV Embossing}

Affordable, large-area nanopatterning on biocompatible films is important for applications such as tissue engineering and gene and drug delivery. Poly(ethylene glycol) patterns have been produced with photolithography, but resolution is limited to about $1 \mu \mathrm{m}$. Electron-beam writing and focused ion-beam (FIB) lithography are both capable of the resolution required for nanopatterning, but both are serial processes (and are therefore timeconsuming) and require specialized substrates. Recently, however, J.X. Gao, M.B. Chan-Park, and colleagues of Nanyang Technological University, Singapore, have developed a practical technique-a combination of FIB lithography and UV embossing — to replicate a nanopattern on a biocompatible polymer.

As reported in the March 23 issue of Chemistry of Materials, Chan-Park and coworkers first created a master mold by using a focused ion beam to etch a nanopattern into a 1-mm-thick titanium nitride (TiN) film. A replication of the master was made using silicone rubber. The silicone casting was then used as the mold for the UV embossing with poly(ethylene glycol) diacrylate (PEGDA) and a photoinitiator. The researchers selected TiN because it has the desired hydrophobicity and durability for multipleuse master molds. PEGDA has been 